\title{
Dustproof cooling of the electrical box
}

\author{
Patrik Nemec $^{1, *}$, Katarína Kaduchová ${ }^{1}$, and Milan Malcho ${ }^{1}$ \\ ${ }^{1}$ University of Zilina, Faculty of Mechanical Engineering, Department of Power Engineering, Univerzitna 8215/1, 01026 Zilina, \\ Slovak republic
}

\begin{abstract}
In present are electrical boxes cooled by air through the intake hole on the bottom electrical box to the box space with electrotechnical elements and exhaust through the hole at the top to the surrounding by natural convection. This cooling method is effective but operate with the risk of contamination electrotechnical elements by dust sucking from surrounding air. The goal of this work is solution of the dustproof cooling of the electrical box by natural convection. The work deal with design of the device with the heat transfer by the phase change of the working fluid and experimental measuring its thermal performance at the cooling electrotechnical elements loaded by heat $1200 \mathrm{~W}$ in the dustproof electrical box.
\end{abstract}

\section{Introduction}

The durability and reliability of electronic devices and electronic components are decreasing with high temperature. When the temperature increases, the parameters of the electronic components change and component can get damaged. This paper deals with heat dissipation problem for the multi-system static converter for a two-storey personal wagon. The static converter is a power electronic device with no moving parts that alter the parameters of electrical energy of one type to other parameters of the desired electrical power of another kind (voltage and current size and time, frequency and number of phases). In the experiment, the Joule heat generated by the static power converter has been simulated by resistive heating elements. Resistive element converts electrical energy into heat through resistance also known as Joule heat. Heating conductor can be explained by forwarding part of the kinetic energy of the particles causing electrical current (typically electrons) to particles, which are not participating in the electric current (usually positive ions in a fixed position). This increases the thermal motion of the particles, and the wire is heated $[1,2,3]$.

The aim of this work is to design and construct a cooling device operating as a thermosyphon loop heat pipe, which would be able to dissipate the heat generated from electronic components, so that their efficiency is to be the highest and the lifespan is to be the longest.

For proper operation of the cooling device, it is important to correctly design evaporating and condensing section. Additional conditions for proper operation of the device are hermetic closures, which contain the working medium (in our case, distilled water) at a defined pressure. The principle of operation is that the evaporator absorbs heat from the environment which causes the working medium inside the evaporator to begin to evaporate at a certain temperature. The steam

\footnotetext{
* Corresponding author: patrik.nemec@fstroj.uniza.sk
}

passes through the steam line into the condensing portion of the loop heat pipe. The condensation part is cooled by the cooling medium (water, air, etc.), which causes cooling of the vapor in the condensing part and condensation of the working medium. Subsequently, the working medium is gravitated to the liquid pipeline and back to the vaporized portion of the loop heat pipe, where it evaporates again. This results in circulation of the working medium connected to the heat transfer. Important factor of cooling device design is the working temperature (max. temperature at which the cooling device will work) of the work medium. Other important factors are location of the heat source from cooling device and method of removal transferred heat by the cooling device (natural or forced convection) $[4,5,6]$.

\section{Design of cooling device}

One of the important parameters for manufacturing and subsequent proper functioning of the cooling device is design of the evaporation and condensing part. The most important parameter in the design is going out of the existing dimensional parameters available to placing the proposed cooling device. The new design is supposed to replace the old method of cooling the power transducer, where electronic devices inside the cabinet have been dusting. The Fig. 1 shows the place of storage of a new cooling device. By changing the phase of the working fluid from the evaporation heat exchanger, which will take waste heat generated by static power converters to the condensing heat exchanger, which will be by forced convection remove transferred heat [7]. 


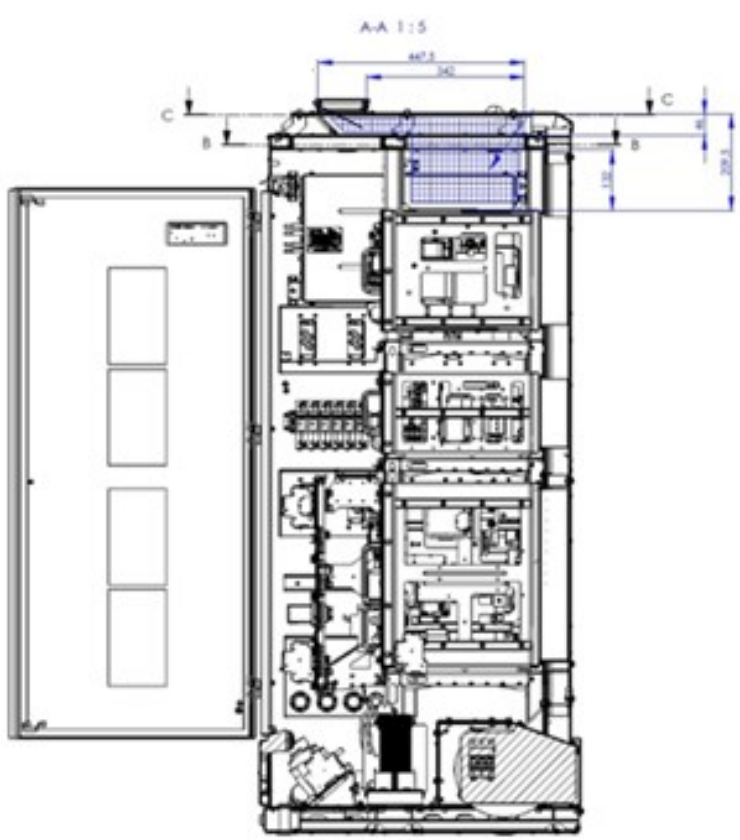

Fig. 1. Cooling device placing in static power converter.

\subsection{Evaporator}

The evaporation heat exchanger is one of two heat exchangers of cooling device. Main focus in designing of evaporator is to achieve the best possible absorption of heat dissipation. The evaporating part in the experimental device consists of two reservoirs connected by a copper pipes. For better absorption of generated heat, the lamellas were designed on the copper pipes. At the bottom of the evaporator is a drainage pipe that connects the two reservoirs. Parameters of the evaporating part of the cooling device are shown in Table 1. In the Fig. 2 and Fig. 3 are views of the evaporating part of the cooling device.

Table 1. Structural composition of cooling device evaporation part.

\begin{tabular}{|c|c|c|}
\hline Lamels & Number & 408 \\
\hline & Dimension & $240 \times 120 \mathrm{~mm}$ \\
\hline & Thickness & $0.25 \mathrm{~mm}$ \\
\hline & Pitch & $2 \mathrm{~mm}$ \\
\hline Pipe & Material & aluminum \\
\hline & Number & 32 \\
\hline & Diameter & $\Phi 16 \times 0.8 \mathrm{~mm}$ \\
\hline & Length & $944 \mathrm{~mm}$ \\
\hline Reservoir & $\begin{array}{c}\text { Dimensions } \\
(\mathrm{t} x \mathrm{v} \times \mathrm{l})\end{array}$ & $120 \times 240 \times 30 \mathrm{~mm}$ \\
\hline & Material & copper \\
\hline
\end{tabular}

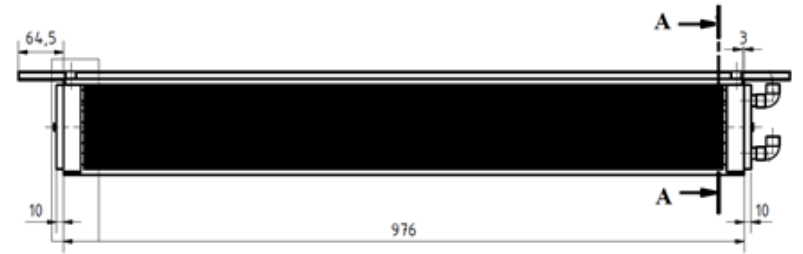

Fig. 2. Evaporating part of cooling device - front view.

$$
\mathbf{A}-\mathbf{A}
$$

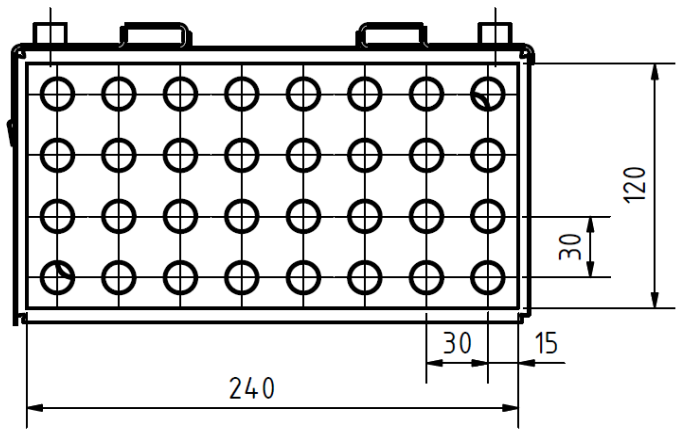

Fig. 3. Evaporating part of the cooling device - side view.

\subsection{Condenser}

The condenser of the cooling device is made of a meander-lined copper pipe with lamellas to increase the heat exchange surface with the surroundings. The condenser is located above the evaporation part. A T-piece fitting is provided on the top of the condensing pipeline for filling working fluid and measuring the pressure inside the cooling device. Parameters of the condensing part of the cooling device are shown in Table 2. In Fig. 4 is a condenser of a cooling device.

Table 2. Structural composition of cooling device condenser part.

\begin{tabular}{|c|c|c|}
\hline Lamels & Number & 332 \\
\hline & Dimension & $420 \times 30 \mathrm{~mm}$ \\
\hline & Thickness & $0.25 \mathrm{~mm}$ \\
\hline & Pitch & $2 \mathrm{~mm}$ \\
\hline Pipe & Material & aluminum \\
\hline & Number & 14 \\
\hline & Diameter & $\Phi 16 \times 0.8 \mathrm{~mm}$ \\
\hline & Length & $785 \mathrm{~mm}$ \\
\hline Fittings & Material & copper \\
\hline & Elbow & R15 \\
\hline & Material & copper \\
\hline
\end{tabular}




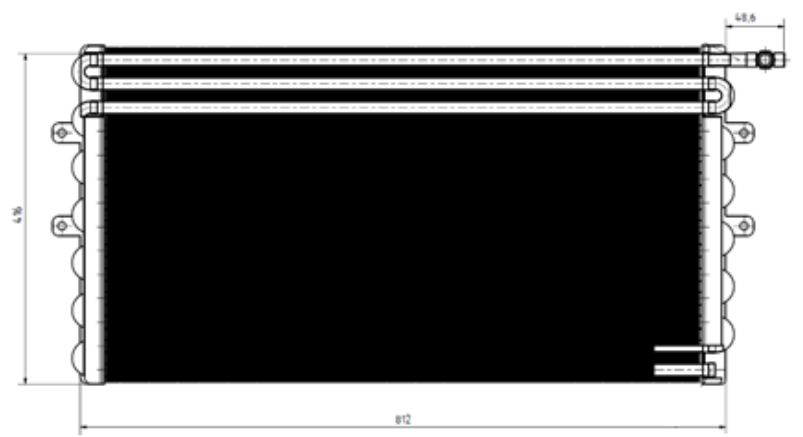

Fig. 4. Condensing part of cooling device - upper view.

\section{Experimental device for verifying functionality and determining the operating parameters of the cooling device}

Verification of functionality and determinating the performance parameters of the cooling device were carried out on an experimental measuring device made from real model of multisystem static power transducer model according to the actual state and is shown in the Fig. 5 and Fig. 6. The model consisted of steel construction of cube profile with dimensions $800 \times 593 \times 1146 \mathrm{~mm}$ (height $\times$ thickness $\times 1$ length). In the steel frame are attached wooden plates of thickness $2 \mathrm{~cm}$. On the back side of the construction is formed profile for connecting air duct to supply air to cool the condenser. Inside cube construction is a wall for the installation of heating devices and to create a space for air flow with the possibility for measuring the temperature at front and behind the evaporator. To reduce heat losses to the environment, the entire experimental device was insulated with $5 \mathrm{~cm}$ thick polystyrene.

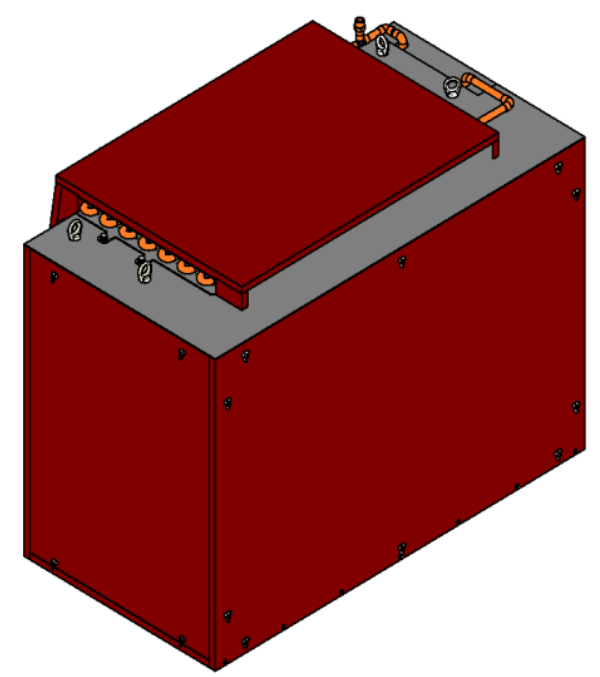

Fig. 5. Experimental device - outside view.

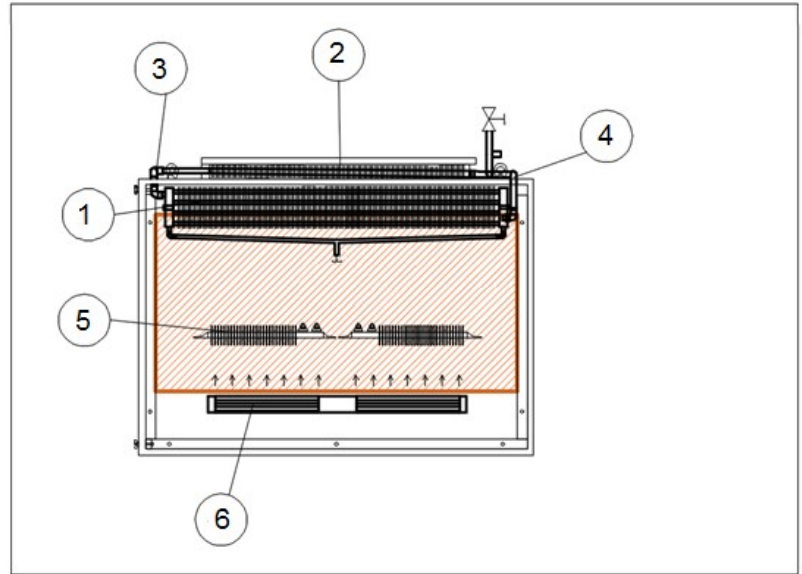

Fig. 6. Experimental device - inside view: 1 - evaporator, $2-$ condenser, 3 - vapor pipeline, 4 - liquid pipeline, 5 - electrical heating element, 6 - tangential fan.

In the Fig. 7 is a schematic connection of the measuring devices to simulation operating condition of electronics elements and to measure operating parameters of the cooling device (amount of the working substance and the cooling air flow rate).

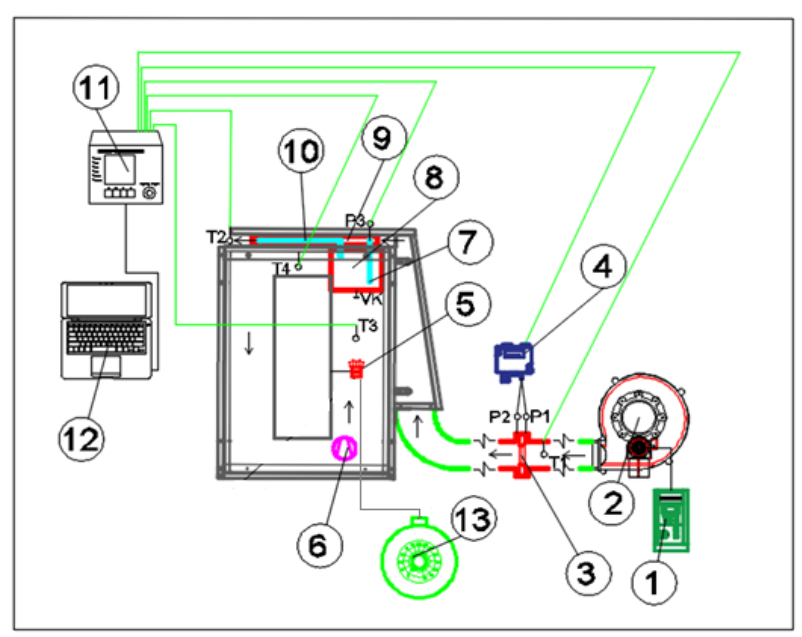

Fig. 7. Schema of entire measuring device: 1 - frequency inverter, 2 - medium pressure fan, 3 - normalized aperture, 4 differential pressure sensor, 5 - electrical heating element, 6 tangential fan, 7 - liquid pipeline, 8 - evaporation part of cooling device, 9 - condensation part of cooling device, 10 vapor pipeline, 11 - data center, $12-\mathrm{PC}, 13$ - regulated autotransformer.

\section{Measurement}

Experimental measurement of the operating parameters of the cooling device was performed at a $1200 \mathrm{~W}$ heat load for a various volume of working media. Heat was generated by two $1000 \mathrm{~W}$ electric heating elements connected in parallel to an electrical power supply (regulated autotransformer) via an active power transducer to define strict value of the electrical power. The tangential fan located inside the multisystem inverter model was designed to better dissipate heat from the heating elements to the evaporating heat exchanger. Cooling of the condensing part was made by the air flow controlled by operating speeds of fan using a frequency 
converter. Measurements were performed at the cooling air flow rate corresponding to operating speed of fan 10 , 20, 30, 40 and $50 \mathrm{~Hz}$. The cooling air flow rate was measured using a standard aperture in the pipeline where a difference in static pressure before and after the diaphragm was scanned by the DPT sensor. For temperatures determining the operating parameters of the cooling device were measured using the Pt100 temperature sensors.

Setting the optimum operating parameters of the cooling device consists of determining the volume of working substance and the rate of cooling air at which the cooling device will most efficiently dissipate heat. The quantity of the working substance is the main parameter for the proper functionality of the cooling device. At small volume of the working substance inside cooling device may entire quantity evaporate and the heat transfer will stop. At high volume of the working substance may liquid phase of working substance overflow entire device and the device will not operate in a two-phase heat transfer mode.

An ideal amount of the working substance in the cooling device is, when it always will have just so much liquid phase in the evaporation part, that consumes all supplied energy at the given conditions to the phase change of its entire volume during its operation.

To determine amount of working fluid in the cooling device, it was assumed that the maximum amount of working fluid corresponded to the entire volume of the evaporator part. The test quantities of the working fluid were determined by the percentage ratios of the evaporator part of the cooling device. The filling of the cooling device was carried out by the method of vacuuming whole volume by the vacuum pump and then filling with a quantity proportional to $20 \%, 40 \%, 60 \%$, $80 \%$ and $100 \%$ of the evaporator volume.

The total volume of the evaporator portion of the cooling device was determined by the sum of the volumes of the individual parts constituting the evaporation part.

$$
\begin{gathered}
V_{\mathrm{V}}=\left(\pi \cdot r^{2} \cdot l\right) \cdot n+(a \cdot b \cdot c) \\
V_{\mathrm{V}}=6.07 l
\end{gathered}
$$

Where $r(\mathrm{~mm})$ is radius of pipes which connect collecting containers, $1(\mathrm{~mm})$ is the length of pipes, $\mathrm{n} \mathrm{(-)}$ number of pipes, a, b, c (mm) are the dimensions of collecting containers.

Distilled water was used as the working fluid in the cooling device. The use of distilled water is in order to obtain a clean working substance free of impurities and minerals which are removed during the distillation process from the water. The change of the liquid phase to vapor phase is determined by operating pressure and temperature evaporating part of the cooling device $[8,9]$.

\section{Evaluation of measured operating parameters}

The calorimetric method was used to determine the amount of power transferred by the cooling device:

$$
Q=m . c .\left(T_{2}-T_{1}\right)
$$

Where Q $(\mathrm{W})$ is the heat flux, $\mathrm{m}\left(\mathrm{kg} . \mathrm{s}^{-1}\right)$ is the mass flow, $\mathrm{T}_{2}(\mathrm{~K})$ is output temperature, $\mathrm{T}_{1}(\mathrm{~K})$ is input temperature and $\mathrm{c}\left(\mathrm{J} \cdot \mathrm{kg}^{-1} \cdot \mathrm{K}^{-1}\right)$ is specific thermal capacity of substance $[10,11]$.

In Table 3 are calculated mass flow rates and cooling air flow rates corresponding to the fan operating speed.

Table 3. Calculation of cooling air velocity.

\begin{tabular}{|c|c|c|c|c|c|}
\hline Frequency & $10 \mathrm{~Hz}$ & $20 \mathrm{~Hz}$ & $30 \mathrm{~Hz}$ & $40 \mathrm{~Hz}$ & $50 \mathrm{~Hz}$ \\
\hline $\mathbf{q} \mathbf{m}\left(\mathbf{k g . \mathbf { s } ^ { - 1 }}\right)$ & 0.0698 & 0.1436 & 0.2140 & 0.2817 & 0.3470 \\
\hline $\mathbf{q}_{\mathbf{v}}\left(\mathbf{m}^{\mathbf{3}} \cdot \mathbf{s}^{-1} \mathbf{)}\right.$ & 0.0601 & 0.1239 & 0.1848 & 0.2429 & 0.2987 \\
\hline $\mathbf{w} \mathbf{~ m . s}^{-1} \mathbf{)}$ & 1.9192 & 3.9569 & 5.9028 & 7.7581 & 9.5402 \\
\hline
\end{tabular}

In Fig. 8 are shown the performance values transferred by the cooling device at different quantities of the working fluid, cooling air velocity at $1200 \mathrm{~W}$ thermal load. According results in the graph are not clear what the amount of the working substance is most suitable for the cooling device, because with change of the cooling air flow rate measured performances are changed too. Based on these results, it is possible to say that at the lower airflow rates, the highest thermal performance was measured.

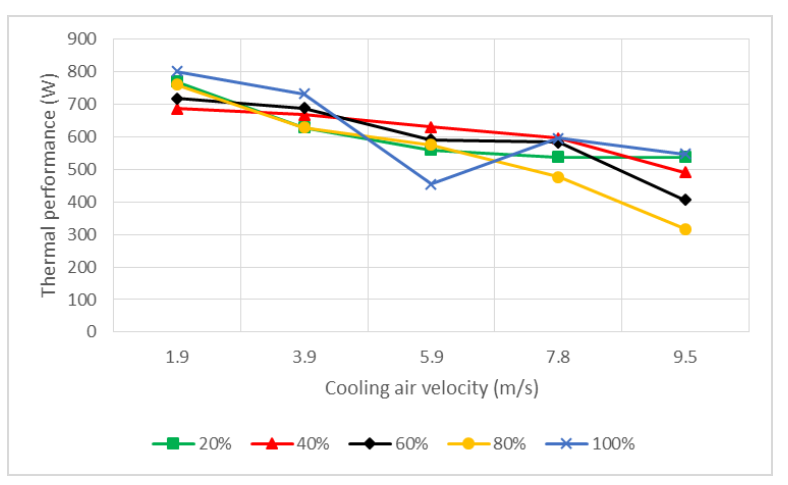

Fig. 8. Thermal performance of the cooling device at different quantities of the working fluid and the cooling air velocity.

Another important indicator during cooling device operation is the ambient temperature measured in front and behind evaporator inside the box with the power electronic components. These temperatures affect the life of electronic components. Lower indoor temperature mean the better operating conditions of the electronic components. When the cooling air velocity was changed, there were no significant differences in the temperatures inside the box. In Fig. 9 are average values of temperatures measured inside the box at individual volumes of the working substance in the cooling device. Fig. 9 shows that increasing volume of the working substance in cooling device increase temperature in the box. 


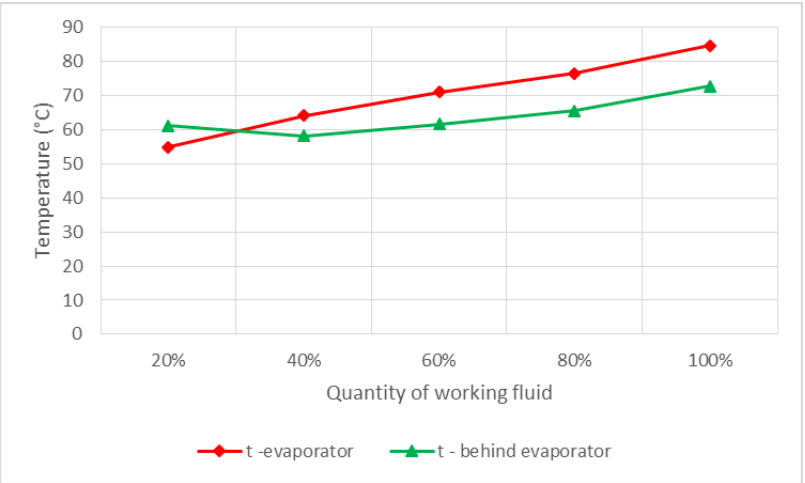

Fig. 9. Temperatures inside the electrical box.

\section{Conclusion}

The aim of this work was to design and construct a cooling device operating as a thermosyphon loop heat pipe, which would be able to remove generated waste heat from the electronic components so that their efficiency was as high as possible and their lifetime was as long as possible. Experimental measurement of the operating parameters of a cooling device with different quantities of the working fluid in the range of 20 to $100 \%$ of the total evaporator volume and cooling air flow rates ranging from 2 to $10 \mathrm{~m} \cdot \mathrm{s}^{-1}$ at a $1200 \mathrm{~W}$ heat load showed that the ideal amount of the working substance in the cooling device is 20 to $40 \%$ and the cooling air flow rate is $2 \mathrm{~m} . \mathrm{s}^{-1}$. At higher volumes of working substance and higher cooling air rates there was a decreasing in heat removal from the surroundings by the evaporating heat exchanger and insufficient heat transfer through the cooling device, resulting in overheating of the evaporator and raising the temperature inside the static power converter model.

This article was created with financial support of the project APVV -15-0778 "Limits of Radiative and Convective Cooling through the Phase Changes of Working Fluid in Loop Thermosyphon" and 042ŽU $4 / 2016$ "Cooling on the basis of physical and chemical processes.

\section{References}

1. P. Hrabovský, Š. Papučík, R. Lenhard, EPJ Web of Conference, 114 (2016)

2. D. V. Kozak, Yu. E. Nikolaenko, International Conference on Electronics and Information Technology, EIT 2016 - Conference Proceedings, (2016)

3. J. Jandačka J. Mičieta, M. Holubčík, R. Nosek, Energy \& fuels, 31, 3 (2017)

4. M. Brůna, Manufacturing technology, 15 (2015)

5. R. Nosek, S. Gavlas, R. Lenhard, V. Sedlak, H. Mollerhagen Arvesen, Communications, 16 (2014)

6. P. Pilát, M. Patsch, Š. Papučík, M. Vantúch, AIP Conf. Proc., 1608 (2014)
7. L. J. Orman, R. Chatys, Transport Means Proceedings of the International Conference (2011)

8. R. Lenhard, J. Jandačka, AIP Conf. Proc., 1558 (2013)

9. J. Cernecky, J. Koniar, Z. Brodnianska, J. of Heat Transfere, 136, 4 (2014)

10. M. Mahdavi, S. Qiu, S. Tiari, Apl. Therm. Eng., 81 (2015)

11. M. Holubčík, P. Durčanský, J. Jandačka, R. Nosek, Scient. World J., 2014 (2014) 